\title{
What might sustainability of the GEO region look like?
}

\author{
Jenny Kingston*a, Leonard Felicetti ${ }^{\mathrm{a}}$, Stephen Hobbs ${ }^{\mathrm{a}}$ \\ ${ }^{a}$ Cranfield University, College Road, Cranfield, UK, MK43 OAL
}

\begin{abstract}
Sustainability in space is often discussed, but as a community we are only gradually learning what it actually means. To inform this understanding, a set of three parallel projects ran at Cranfield University (Oct 2020 to Mar 2021) to develop a scenario of sustainable use of the geostationary orbit region. The three projects were to develop mission designs for (a) a Scavenger spacecraft equipped with tools, actuators and sensors to perform rendezvous with selected satellites at their end of life, to harvest selected parts and components (i.e. solar panels, radiators, antenna reflectors), store and deliver them to the Recycler for refurbishment or recycling, (b) a Recycler space station located in GEO, capable of receiving parts and materials obtained by the Scavenger spacecraft and performing a range of inspection, recycling and repurposing operations on them, and (c) a candidate customer mission: a huge communications satellite based on the Airbus VASANT (VASt ANTenna) concept, with two antenna arrays, each $35 \mathrm{~m}$ square, sized to be able to communicate directly from GEO to mobile phone users at Earth's surface.

Some of the features highlighted by these studies are (a) the technical challenges of reusing parts from old satellites: modularity and design-for-reuse seem to be key enablers, (b) the advanced robotics and autonomy implied by the on-orbit operations, (c) the challenge of long-term orbit control without excessive propellant consumption, and (d) although the technology is challenging, there are major non-technical challenges for the business case and for aspects such as the legal use of debris, liability for accidents, and compliance with any regulations. Sustainability is challenging, but nature leaves us no alternative.
\end{abstract}

Keywords: GEO, sustainable, recycling, salvage, VASANT

\section{INTRODUCTION}

Sustainability in space is often discussed, but as a community we are only gradually learning what it actually means. It has so far largely been considered in the context of mitigation and remediation of space debris, to assure our ability to continue to use near-Earth space safely in the future. Mitigation aims to prevent accumulation of new debris objects, by implementing end-of-life removal plans for new spacecraft being launched. Remediation aims to actively remove the existing debris objects that are most likely to generate further hazardous debris. More recently, initiatives such as ESA Clean Space have widened this to consider the environmental impact of the whole life cycle of a mission, including terrestrial production activities and the launch phase, as well as on orbit [1]. As is becoming increasingly standard practice in terrestrial industries, considering the whole life cycle implies concern for the end-of-life of an item; where possible the aim is to reuse, repurpose or recycle, rather than merely dispose of it. This is the circular economy concept, as opposed to the conventional linear economy production model of "take, make, dispose" [2]. It can be considered that even a successful implementation of space debris mitigation and remediation is still operating within the linear economy model, as the debris is just being disposed of. It no longer causes harm, but it is not providing any benefit. In fact, the disposal implies further resource costs (financial and material, since remediation requires further spacecraft launches just to remove the debris objects).

This study considers a scenario for a more sustainable use of Geostationary orbit, a particularly valuable region of space. The core underlying concept is to view debris objects in this region as a resource to be used, rather than a threat to be removed. The debris will be reused, repurposed or recycled, creating a basis for a circular economy in space. Whilst recycling of space debris is not itself a new concept [3][4], here it is considered what would be required to enable such an ecosystem in GEO.

*j.kingston@cranfield.ac.uk 
The scenario we propose here is a first step towards a vision for GEO sustainability. We recognise that it is neither complete nor fully-optimised, but we believe that it already provides useful insights into space sustainability.

The article next introduces the concept of a sustainable space ecosystem. Each of the principal components of such an ecosystem is then described, followed by a discussion of the lessons learned from this study. We close with some final remarks and suggestions for further study.

\section{SUSTAINABLE SPACE ECOSYSTEMS MISSION CONCEPT}

To inform this study, a set of three parallel MSc student Group Design Projects ran at Cranfield University to develop a scenario of sustainable use of the geostationary orbit region. Geostationary orbit was selected as the location for several reasons. Although it has a much lower debris population than the other Protected Region, Low Earth Orbit, it is cheaper in delta- $\mathrm{V}$ terms to move between debris here (due to the similar altitudes and inclinations), and it was considered there is more likely to be a significant commercial customer base in this orbital region.

The projects developed mission designs for (a) a Scavenger spacecraft to harvest useful parts from satellites at their end of life, (b) a Recycler spacecraft to process the scavenged parts into usable items, and (c) a candidate customer mission (a huge communications satellite based on the Airbus VASANT concept).

The Scavenger is equipped to perform rendezvous with selected targets, harvest parts and components (initially solar panels, radiators, antenna reflectors), store and deliver them to the Recycler for processing. Selection of the targets is made among the population of non-operative satellites in GEO, and manoeuvres are planned in order to minimise the propellant consumption and consequently maximise the number of accessed satellites. The operations shall minimise the risk of collisions between the Scavenger and the target satellites but, even more important, both Scavenger and Recycler must avoid the release of further space debris while accessing the components.

The Recycler is located in GEO, and capable of receiving parts from the Scavenger spacecraft and performing a range of inspection, recycling and repurposing operations on them. Scavenged materials can be re-formed into structural elements or reused in their original form if inspection shows they meet a minimum performance threshold. Suggested useful outputs were defined based on the requirements from the customer, VASANT. Further onboard processes are proposed to produce coatings for new radiator tiles, and harvest propellant to allow for self-sufficient East-West station-keeping over an extended lifetime.

The Customer: In general terms this will be any entity with an interest in procuring parts or components for on-orbit assembly of space infrastructure. In the example here, the case of VASANT is used. This is a VASt ANTenna communication satellite, sized (with two antenna arrays, each $35 \mathrm{~m}$ square) to be able to communicate directly from GEO to terrestrial mobile phone users. For this study we assumed that an upgrade to VASANT was required to increase its capacity by $50 \%$, and that for sustainability this would be done using as much recycled hardware as possible. An associated Tug spacecraft to collect parts from the Recycler, and a Servicer robot to upgrade and service VASANT (and the Tug) were also proposed. For sustainability we assumed (1) that there should be no long-term growth of the mass in GEO, so all parts either had to be recycled or had a plan in place for their removal or reuse at end of life, and (2) that the spacecraft's service life should extend well beyond the standard 15 years.

It is important to note that all three players are required for a successful circular economy, and there must be a significant degree of concurrent design and development. As shown in Figure 1, without the presence of customers, the recycling station and scavenger are redundant. Without a good understanding both of what the recycler may be able to provide and what this may enable a customer to build, there will be inefficiency and lost opportunities, and little chance of economic feasibility. 


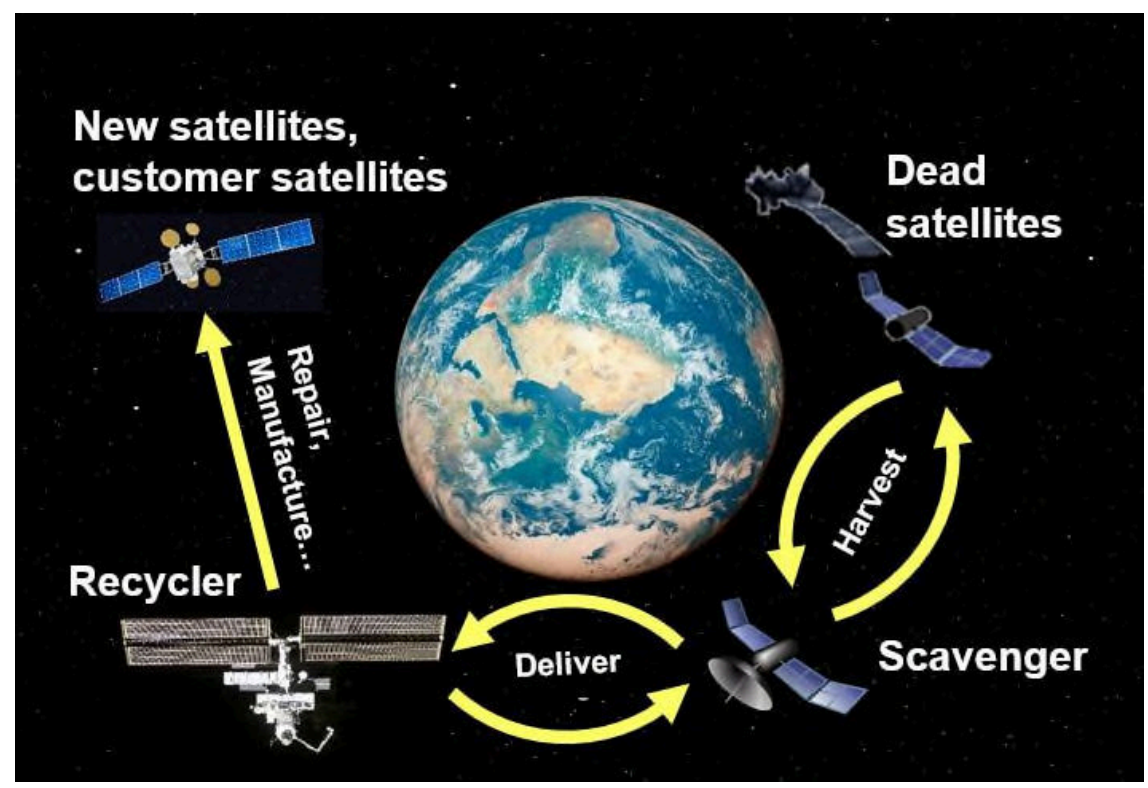

Figure 1. Sustainable space ecosystem mission concept.

There are several considerations which provide a rationale for such a concept:

1. Launch: High cost to deliver satellites to GEO. Launch limitations in terms of mass and (perhaps more importantly) volume. Volume restrictions require complex deployments to allow large structures such as antennas, solar arrays, radiators etc to be used. High launch costs make items produced on-orbit more valuable; conversely lowering launch costs reduces their value and tends to undermine the purely financial case for recycling.

2. Enabling of new applications: Interest in use of very large spacecraft to enable new types of commercial services. Very large satellites such as VASANT would require on-orbit assembly as they could not be delivered in a single launch.

3. GEO debris population: Existence of many largely intact dead satellites in GEO and its vicinity, which tend to have relatively similar architectures. This would allow a more standardised set of capabilities to be defined for a scavenger, which still allow it to target a large number of debris. The debris is accessible at relatively low delta$\mathrm{V}$ cost. It is also a region of high commercial use.

4. Current/emerging technology: Technology developments in proximity operations, rendezvous and docking, and robotic interaction/manipulation, which are being increasingly demonstrated via on-orbit servicing and active debris removal missions. There are also developments in on-orbit manufacturing and assembly. These provide many of the functions which would be required for successful scavenging of debris.

The following sections describe the concepts developed for each player within the mission scenario.

\section{DEBRIS SCAVENGER SPACECRAFT}

The main function of the Scavenger spacecraft is to harvest materials and components from defunct satellites in GEO, store and deliver them to the recycler space station. For this reason, its design was strongly driven by requirements imposed by the Recycler, especially about the typology of the components that need to be collected. After an initial survey of the existing and future dead satellite in GEO, an assessment of the actual abundance of specific components, also considering the complexity of harvesting/dismantling and eventual availability of processing technologies for repurposing and/or recycling materials, it was decided that the most suitable components to be harvested are:

1) Solar Arrays. The typical configurations of GEO satellites have large solar panels that can be easily dismantled because generally located outside the main bus of the satellite. Even if not totally efficient at EOL, these can be reused after being refurbished by the recycling station.

2) Antennas. They are generally made of composite material that can be reprocessed/melted to obtain structural elements. They are relatively easy to be dismantled, as they are outside the main body of the spacecraft. 
3) Radiators. They are the external panels of the satellites, covered by radiative coatings and connected to the internal devices either through conductive couplings or heat pipes. The composite material can be reprocessed to obtain structural material and, the ammonia in the heat pipes can be reused as a propellant for the thrusters of the Recycler.

The Scavenger spacecraft shall indeed perform all the required operations to identify, dismantle, manipulate, and store such components. Robotic arms seem the most suitable tools for performing such operations, and consequently, the design of the spacecraft reflects this design choice. Figure 2 shows the baseline configuration of the Scavenger in both the fully deployed and loaded configurations. The frontal robotic arms carry the eye-in-hand cameras and cutting-lasers used to closely observe the target components and eventually cut them out from the target satellite, respectively. Other robotic arms are used to catch and manipulate parts of the target satellite during the harvesting operations and eventually hold the dismantled parts once they are completely disconnected from the satellite's main body. Finally, ad-hoc designed clamping mechanisms are used to collect and store the scavenged components during the orbital transfers, as shown in Figure 2.b.

The target components to be scavenged are all easily accessible from outside the spacecraft. Therefore, there is no need for complex and dexterous manipulation systems to perform surgical-like operations inside the target spacecraft. All the target components can be removed by using cutting tools. The selection of such tools considered different options, such as motorised saws, hydraulic shears, and lasers. These options were traded off in terms of cutting performance, risk of producing additional debris and damaging the components, TRL, mass, complexity, power required, thermal influence, reliability, and cost [6]. As a driving requirement, the study considered that the preferred tool shall guarantee that no additional debris is produced during the cutting operations. If some particles are produced, these are small enough not to damage other spacecraft in the GEO region. Laser cutting tools were consequently selected as primary tools as they can be used to harvest all the target components while guaranteeing a sufficient degree of cleanliness of the operation compared to other tools (less mass ejected during the cutting operations).
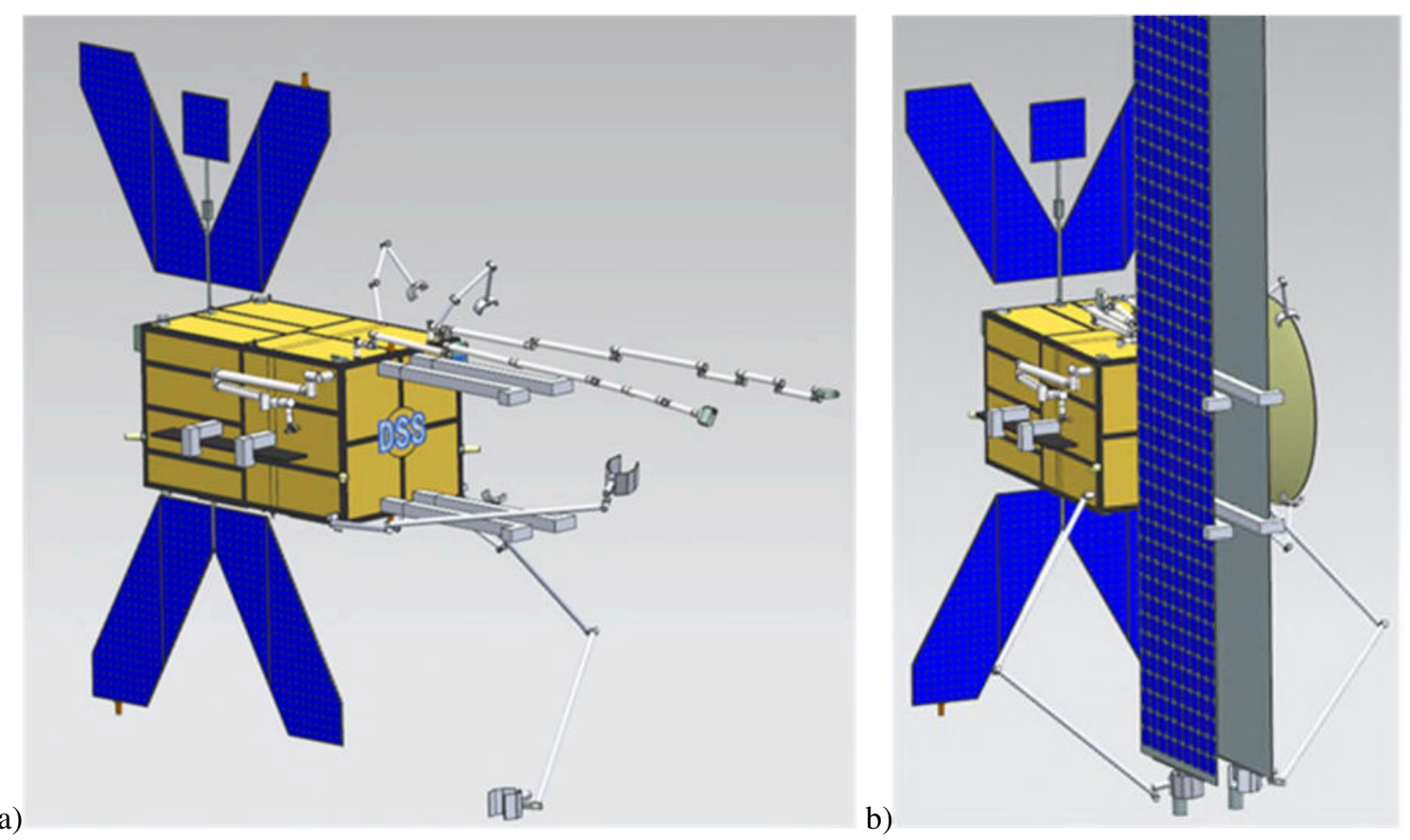

Figure 2. The Debris Scavenger Spacecraft (DSS): a) fully deployed and b) fully loaded configurations.

To guarantee the success of the harvesting operations, it is necessary to have a stable and solid connection between the Scavenger and the target satellite. Rendezvous and grasping represent critical but important phases for the success of the operations of the Scavenger spacecraft. These operations are performed using one of the robotic manipulators equipped with a gripper designed to grasp the launcher adapter rings. Indeed, launcher adapter rings represent a solid and almost standardised interface for all the satellites in the GEO region. The relative navigation tool used during the close approach to the target is based on a Tridar system [7]. In order to have enough control authority during the capture and de-tumbling phases, the Scavenger spacecraft is equipped with $2 \times 12 \mathrm{MMH} / \mathrm{MON}-3$ thrusters that guarantee 6 degrees of freedom 
movements to the spacecraft. Reaction wheels, a standard suite of attitude sensors, alongside a Xenon-based electrical propulsion system, are used during the transfer phases between the Recycler and the target satellites [8].

The adopted concept of operations for the Scavenger mission is illustrated in Figure 3. After launch and insertion to the GEO region, the Scavenger spacecraft will need to go back and forth from the GEO orbit to the graveyard orbit targeting each time a different dismissed satellite. Even if more convenient in terms of delta- $\mathrm{V}$, the option of having a journey targeting multiple satellites per travel was discarded, as it would have required an increase of the mass and dimension of the Scavenger to host additional storing slots for the scavenged components.

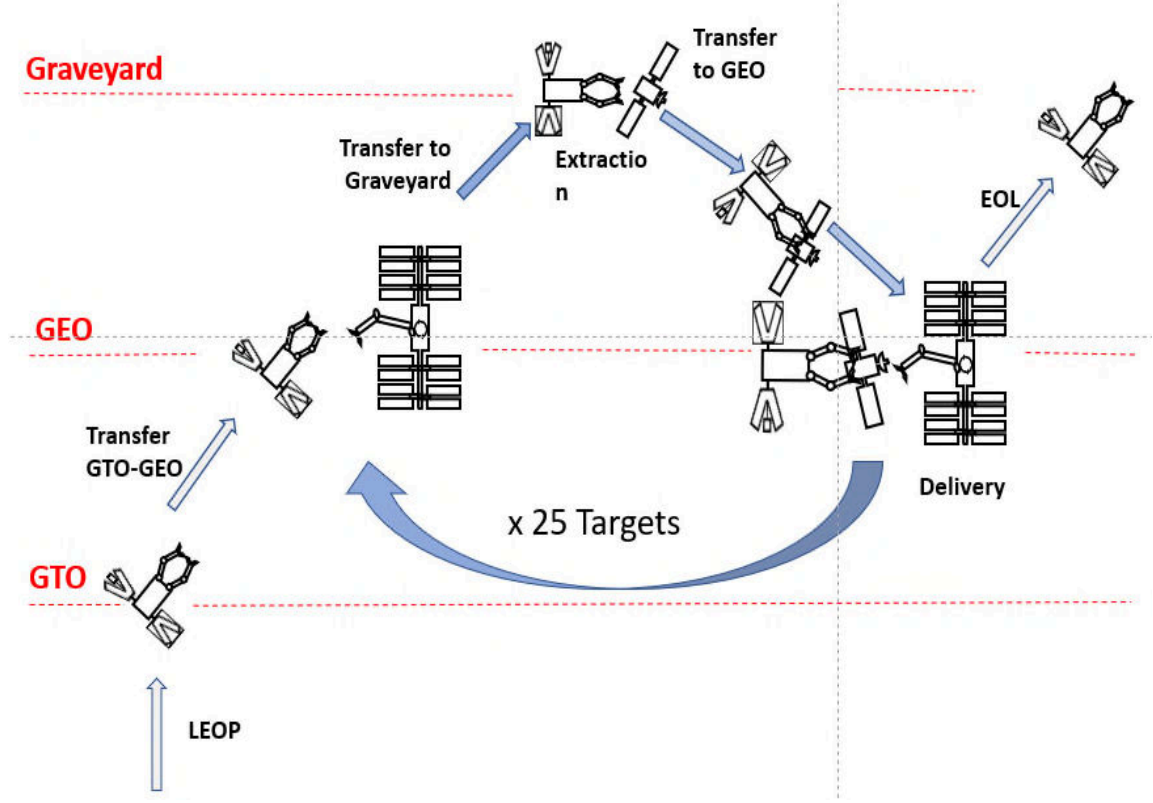

Figure 3. Concept of operations for the Debris Scavenger Spacecraft

The selection of the transfer strategy considered the constraint imposed by the fixed GEO position of the Recycler and the evolution of the orbital positions of the target satellites due to orbital perturbations. The graveyard orbit is indeed strongly affected by lunisolar effects producing changes of inclination of the orbit up to $15 \mathrm{deg}$. The strategy accounts for delta-V for performing the GEO-Graveyard transfers, including eventual orbit plane changes and drifts to cancel out the phase difference between the Scavenger and the target satellites. Depending on the inclination and altitude of the given target, the cost in terms of delta-V can vary from $42 \mathrm{~m} / \mathrm{s}$ to $216 \mathrm{~m} / \mathrm{s}$ per each leg of the travel [9]. It is assumed that the Scavenger shall operate for at least 10 years targeting 25 selected satellites. Orbit insertion, disposal and final rendezvous maneuvers were also considered in the investigation. As a result, the propellant mass necessary to support the operations was estimated as $2434 \mathrm{~kg}$, that added to the $2941 \mathrm{~kg}$ of the spacecraft's dry mass, gives a final wet mass of about $5475 \mathrm{~kg}$. In its stowed configuration, the Scavenger's size is $2.6 \times 2.6 \times 4.5 \mathrm{~m}^{3}$ allowing for being launched by Ariane 6 .

\section{RECYCLER SPACE STATION}

The Recycler Space Station concept defined in this study is modular in architecture. Due to its large size, it would require multiple launches and use of on-orbit assembly. The overall architecture is shown in Figure 4. The Recycler modules comprise:

Manufacture and Testing (MAT module)

This module contains the equipment for initial inspection and handling of incoming debris materials. It includes a laser cutter to allow sheet materials (solar panels, antenna reflectors etc) to be cut into standard-sized tiles for further processing. The main propulsion unit is also situated here.

Power and Storage (P\&S module)

This module contains the transfer mechanism to deliver materials from the MAT to the recycling unit, and hosts the Canadarm robotic arm which accepts incoming materials from the Scavenger. Materials can also be stored within this 
module. It also houses the solar arrays and batteries, and the TT\&C equipment. The P\&S module will be the first to be launched, and the Canadarm will allow for assembly of the Recycler station, as well as grappling of Scavenger and customer spacecraft. Grapple fixtures on the other modules allow the arm to move around as required, to enhance accessibility and handling capabilities.

Advanced Recycling Operations (ARO module)

This is where the major recycling activities take place. Within this module, different units separate materials, melt aluminium for Metal Injection Moulding of new components, pyrolyse composite materials to produce gases for use as propellant, and process re-used structural materials into radiator panels. Waste material that cannot currently be usefully recycled is also collected here for storage and later transfer to the GEO graveyard as required. Future improvements in recycling technologies aim to reduce the quantity of waste material as far as possible. It could also potentially be used in the interim as shielding (from radiation and/or debris).

External Storage (ES module)

Finally, large components (either newly-manufactured or items incoming from the Scavenger) can be stored externally on this module.

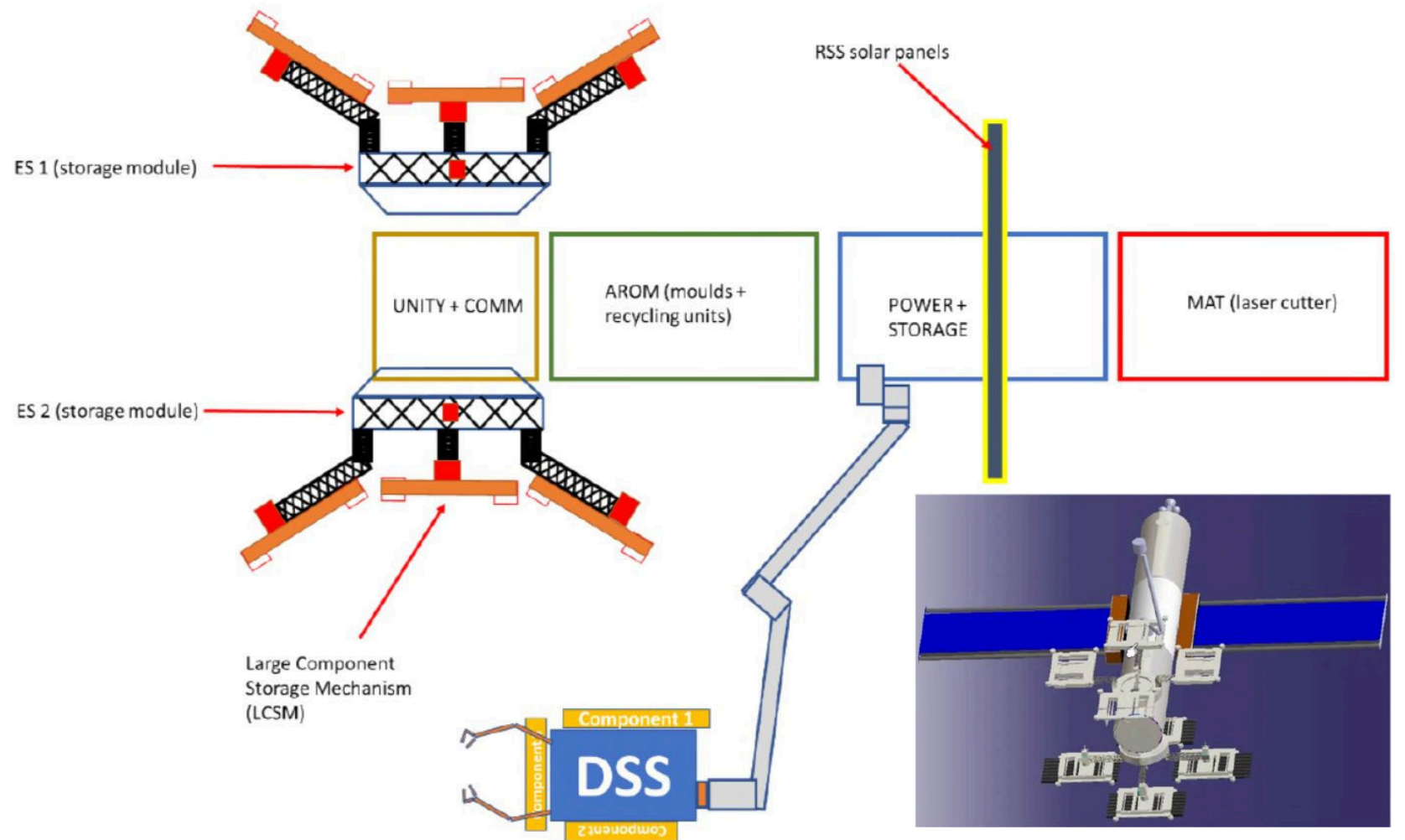

Figure 4. Architecture of the Recycler Space Station (RSS), showing grappling with the Debris Scavenger Spacecraft (DSS) delivering recovered debris items and (inset) a 3D view.

The outputs produced by the Recycler have been informed by requirements provided from the VASANT mission. These outputs are:

- $\quad$ Struts - these are produced by metal injection moulding from scavenged aluminium, as hollow tubes. Connection fittings are also produced to allow large structures to be assembled.

- Solar panels - these are scavenged intact, tested and if found to meet a defined performance threshold, they are reconfigured for use on VASANT.

- Radiator panels - these are again scavenged, and coatings applied via vapour deposition of aluminium. 
In addition, the Recycler makes use of scavenged ammonia (from heat pipes in the radiator panels of dead satellites) in arc-jet thrusters for its propulsion. Scavenged structural panels can also be cut and re-formed into new structural shapes, using connectors produced from recycled aluminium.

The new parts are stored until they can be collected by customers. In this example, the VASANT customer sends a tug vehicle to collect the parts and deliver them to the VASANT location.

The Recycler is proposed to be placed in GEO, at a longitude around $150^{\circ}$ West, selected due to its lower desirability for communications purposes (reducing competition for this longitude slot), its footprint being mainly positioned over the Pacific. Position in GEO gave the significant advantage of both convenience to future customers, and a constant communication link with a single ground station positioned within the station's footprint (on Hawaii), considered essential due to the complex processing and handling operations being conducted onboard. It was also found that there is a significant population of dead satellites that remain in GEO rather than GEO graveyard (861 objects, of which 761 are intact satellites). It was decided to perform only East-West station-keeping, and allow the inclination to drift. This greatly reduced the requirement for station-keeping delta- $\mathrm{V}$ and hence propellant. Without performing inclination correction, it is foreseen that enough ammonia could be scavenged to meet the station-keeping propellant needs without resupply from Earth.

The mass budget and dimensions for the Recycler, by module, is shown in Table 1. Each module is launched into Geostationary Transfer Orbit on a separate Falcon Heavy launcher, and carries its own Apogee Kick Motor for orbit insertion. The station uses Roll-Out Solar Arrays capable of producing $55.9 \mathrm{~kW}$ at minimum end-of-life (15 years).

\begin{tabular}{|l|l|l|l|}
\hline Module & Quantity & Launched Mass (tonne) & Dimensions \\
\hline MAT & 1 & 16.2 & $\varnothing 4.5 \mathrm{~m}$, length $10 \mathrm{~m}$ \\
\hline P\&S & 1 & 22.2 & $\varnothing 4.5 \mathrm{~m}$, length $10 \mathrm{~m}$ \\
\hline ARO & 1 & 28.5 & $\varnothing 4.5 \mathrm{~m}$, length $10 \mathrm{~m}$ \\
\hline ES & 2 & 18.9 & $\varnothing 3 \mathrm{~m}$, length $8 \mathrm{~m}$ \\
\hline
\end{tabular}

Table 1 Recycler module masses and dimensions

The concept of operations and the overall programme lifecycle of the Recycler is shown in Figure 5. After the initialization phase, where each of the modules is launched and on-orbit assembly takes place, the first recycling phase starts in 2032 , in tandem with the first Scavenger spacecraft. This phase lasts 5 years and operates as a proof-of-concept, building up capability, providing client services on a smaller scale and starting to prepare the outputs required by VASANT. From 2037, production is ramped up, with two further Scavenger spacecraft in place, to support the VASANT upgrade programme.

From 2042, the station provides on-going supply to VASANT, as well as to other customers. Upgrade of the station is envisaged during this phase, to allow more complex recycling and manufacturing operations, and to store more parts. After 2047, the intention would be to further upgrade and expand the station, incorporating new technologies and working with a new generation of Scavenger spacecraft capable of targeting and processing a wider range of targets and components. 


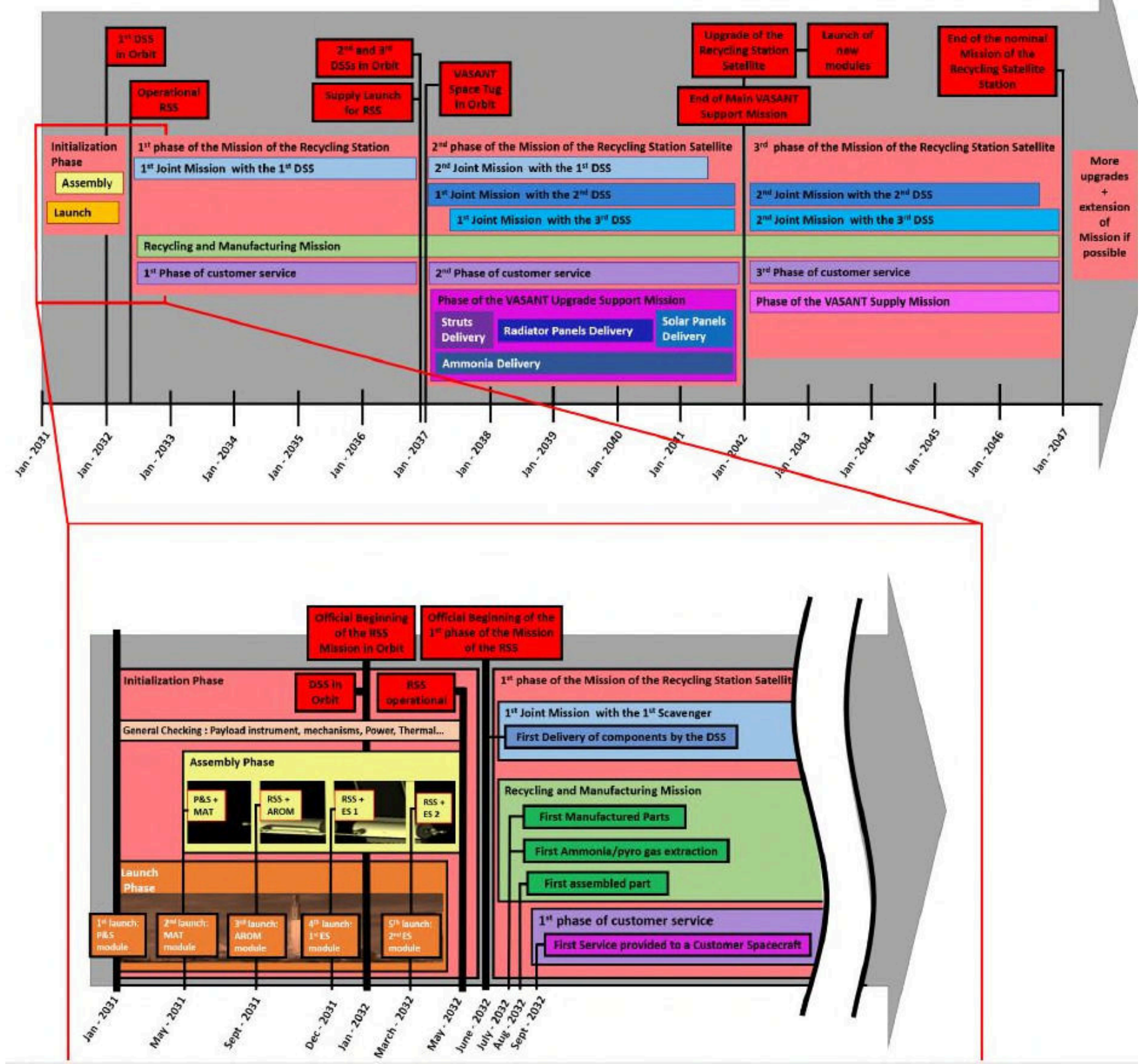

Figure 5 Concept of Operations for the Recycler Space Station

\section{CANDIDATE USER: VASANT}

As a candidate customer for the Scavenger and Recycler facilities, we used the VASANT concept [5] for a huge communications satellite in GEO. Figure 6(a) shows a version of the original concept. The driving user requirement is the need to communicate directly from GEO to mobile phone users on Earth's surface: hence the need for very large antennas (antenna arrays are approximately $30 \mathrm{~m}$ in size). For this study we used a modified version of the original VASANT concept and assumed that it was to be upgraded by increasing its total area by $50 \%$, hence there are three antenna arrays rather than two in Figure 6(b). The total mass of VASANT 2 is about 100 tonnes (including margin).

In addition to an upgrade, we assumed that the mission should be compatible with a strict sustainability rule which permitted no long-term increase in the mass in the GEO region. Hence any mass required for VASANT2 either had to be 
removed from GEO at the end of the mission (as an integral part of the mission concept) or had to be recycled from existing assets in GEO. In consultation with the Recycler team, we assumed that solar arrays, some propellant, and some structural elements could be provided by the Recycler: the rest of the mass had to be delivered from Earth's surface and then disposed of at the end of VASANT2's operational life.

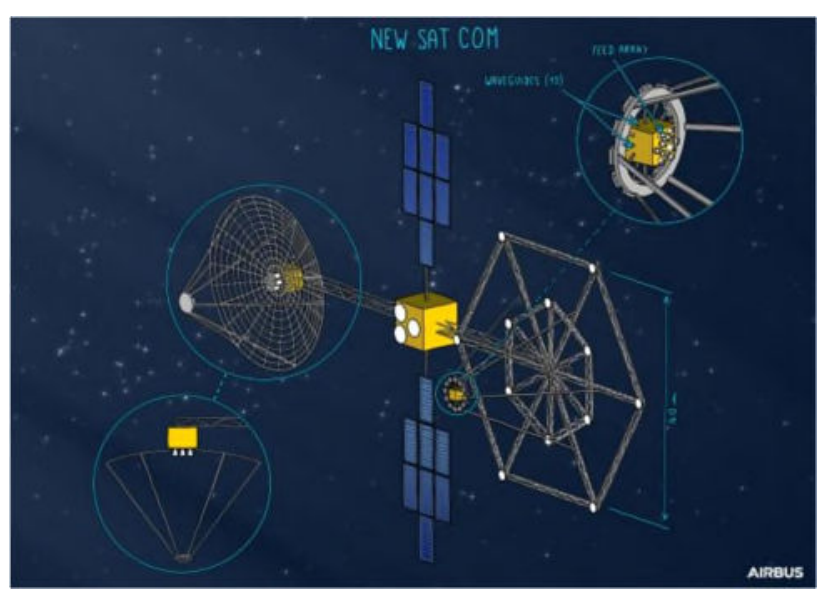

(a) Original VASANT concept [5]

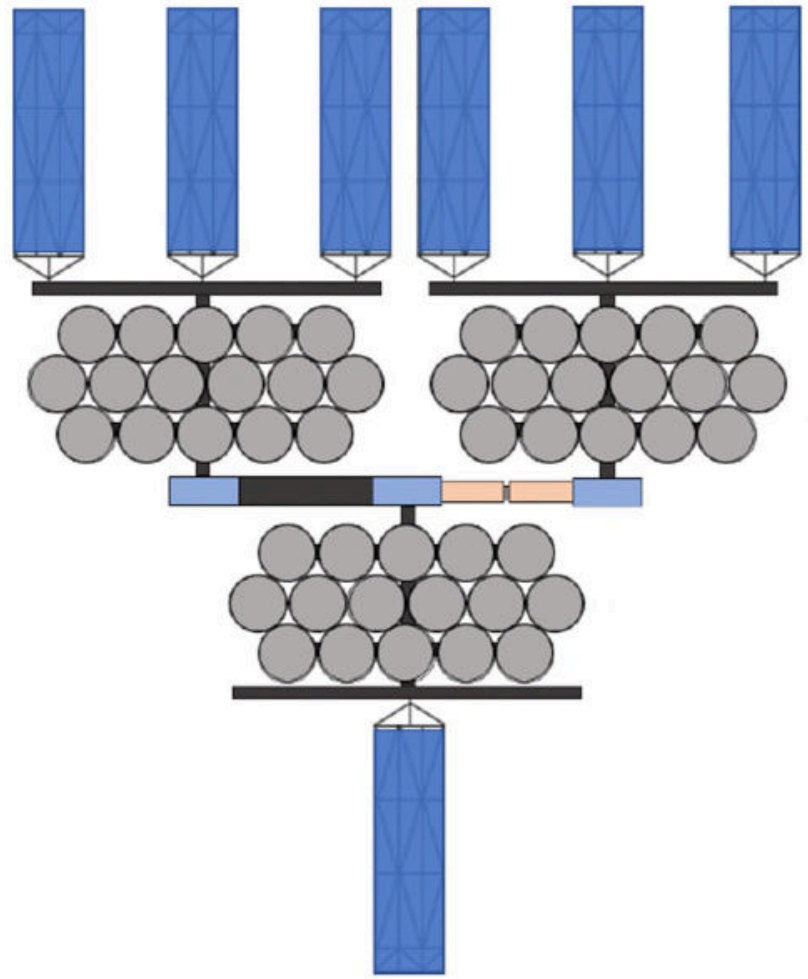

(b) Concept for an upgraded VASANT (VASANT2) [11]

Figure 6. Two versions of the VASANT concept for a huge geostationary communications satellite

In addition to VASANT2, we assumed two other spacecraft for the mission concept:

- Tug to transport components from the Recycler to the Servicer working on VASANT2 and from the launch vehicle to the Servicer,

- Servicer to upgrade VASANT to VASANT2, and to service the Tug, VASANT2, and itself (Servicer crawls across VASANT and is not free-flying).

\begin{tabular}{|l|l|l|}
\hline Component & Dry Mass (tonne) & Wet Mass (tonne) \\
\hline VASANT2 & 96.2 & 98.4 \\
\hline Tug & 2.69 & 3.99 \\
\hline Servicer & 3.65 & \\
\hline
\end{tabular}

Table 2. Outline mass budget for the VASANT2 mission (all values include a design margin of 20\%) [10]

Although this requirement has not been fully analysed, we assumed that VASANT should be designed to have a long lifetime to improve its sustainability. We therefore envisaged a range of lifetimes before replacement for sub-systems:

- Structure should function for 50+ years before needing replacement,

- Antenna reflectors should be refurbished every 25 years, 
- Electrical and thermal infrastructure (power generation and distribution, thermal dissipation) should be refurbished every 15 years,

- Electronic equipment should be replaceable every 5-10 years so that VASANT's payload can remain compatible with the evolving market demand.

Orbit maintenance has not been fully analysed since it must satisfy user requirements as well as operational constraints and be compatible with the recycling infrastructure. Mobile phones have omnidirectional antennas and therefore the orbit inclination could be uncontrolled (inclination would vary $0-15^{\circ}$ cyclically, with a full period of $\sim 52$ years). However, to meet ITU regulations its longitude excursions may need to be limited (incompatible with free inclination drift), and for low delta- $\mathrm{V}$ transfers to and from the Recycler it may be necessary to control inclination. We assumed that VASANT was kept with near-zero inclination. This "costs" $50 \mathrm{~m} \mathrm{~s}^{-1} \mathrm{yr}^{-1}$; for propulsion Isp of $1000 \mathrm{~s}$ this corresponds to an annual propellant mass consumption of $0.5 \%$ of the mass of VASANT (and is inversely proportional to Isp). This propellant could be supplied from Earth: after use it does not remain in GEO and so does not compromise sustainability.

A concept like VASANT requires on-orbit assembly, since it is far larger than any single launcher could accommodate, and requires multiple launches to GEO. To minimise the mass added to GEO it is desirable to recycle as much mass as possible within GEO to build VASANT2. From a user's perspective, the recycled components (e.g. solar array modules) should ideally be provided in standard sizes and with common interfaces, with guaranteed performance, and be available with an assured delivery schedule known well in advance (at least a few years): these are challenging requirements for the on-orbit recycling infrastructure.

\section{LESSONS LEARNT}

The presented scenario represents a first step towards addressing in an innovative and rational way the problem of recycling of space debris in GEO region. We believe that the interaction among different student groups and the intrinsic multidisciplinary nature of the project, challenged the students in a constructive way, offering numerous reflection opportunities and highlighting possible challenges for a future implementation of such a scenario. These studies pointed out some open challenges and existing technological gaps that still might represent limiting factors for the effective implementation of sustainable space. We want to share these considerations with the aim of opening a constructive discussion on the most promising path for addressing such issues, and build a cleaner and more sustainable space ecosystem in the long run.

For the Recycler, there will be a need to assess, and demonstrate, the feasibility of adapting terrestrial recycling processes to the space environment. Key challenges here are microgravity, power limitations, autonomy or teleoperation, and the extreme difficulties in cleaning and servicing/repairing/replacing equipment. It must also be noted that the concept of value of recycled materials may be very different in a space context. Developments in terrestrial recycling may focus on recovery of materials with high intrinsic value, such as precious metals. However, in a space context there could be considered to be potential value in any material in orbit, simply coming from the investment made in getting it there. The challenge is to find a way to make appropriate use of it in situ. There is likely to be some degree of mismatch between technologies developed for terrestrial use, which will focus on the most useful and economically viable materials to recycle and reuse in that context, and the technologies that will be most suited to utilising scavenged materials in space.

Another challenge for the Recycler is that a lack of inclination control will cause issues of accessibility by customers over time, as the inclination increases. Although there is likely to always be debris available in a matching inclination, so there would not necessarily be an issue for the Scavenger-Recycler system, customers in equatorial GEO will be unwilling/unable to access the station due to the high delta-V required for the inclination change.

From an economic perspective, the Recyling station raises significant questions. The station would require 5 Falcon Heavy launches, and the overall mission cost has been estimated to be in the order of $\$ 25 \mathrm{~B}$. It is difficult to envisage such a mission being financially viable as a purely commercial enterprise; the wider environmental benefits would certainly also need to be considered, and probably would in fact be a more important factor, at least in the short term.

The design of the Scavenger spacecraft considered and targeted the current population of objects populating the GEO. Such satellites are not meant to be harvested or dismantled in orbit, and this represented an intrinsic limiting factor for the project. Only few parts of the current typical GEO satellites can be effectively dismantled in orbit without the risk of 
producing additional debris. The design process also highlighted that the kind of operations and the selection of tools for accessing and removing components were the main drivers for the spacecraft design. The investigation raised concerns on the actual success of the scavenging operations with the current space-qualified robotic technologies: a gap in the dexterity and required autonomy of robotic systems to perform such operations was identified as critical for the mission success. The solutions adopted in the project reflect this issue, as only simple operations such as laser cutting, grasping, and extraction of external components were considered sufficiently safe and realistically implementable in space. Disassembling the components in the reverse way they were built was another option considered for the component extraction. This type of operation, though more complex, may be feasible provided that detailed information about the target configuration and manufacturing can be acquired from the target satellite owners. The main problem arises when satellites are built by different manufacturers, as they would have little to no modularity or standardised ways of assembling components. These issues remarked the importance of having standardised interfaces when designing and integrating the next generations GEO satellites. More in general, a shift of the design paradigm towards the "design to be recycled and reused" would be a stepping stone for building a more sustainable space ecosystem.

The actual availability of propellent onboard is limiting the lifetime of the scavenger mission. The current baseline considered that 25 targets could be reached within 10 years, which was limited mainly by launch constraints. The option of in-orbit refuelling was explored but then discarded as a dedicated one-off transport vehicle was necessary, creating additional debris to be removed and making the system and the operations of the Scavenger spacecraft even more complex. The production of propellant from the recycled (composite or metallic) materials in orbit would represent a game-changer in this scenario, but the current TRL of the technologies for the production and utilisation of recycled forms of propellant is still low.

Another problem was identified on the actual disposal strategy to be adopted for the Scavenger spacecraft at the end of life. This is indeed an additional object inserted in orbit that would increase the population in the GEO region. In the ideal scenario, the same Scavenger spacecraft might be built by using the "design to be recycled and reused" philosophy. Thus, becoming a suitable and preferable target for a new Scavenger mission and consequently contributing to the circular reutilisation of the resources already inserted in orbit.

The VASANT mission design raises questions about such a concept as well as pointing to technology developments which could significantly impact its feasibility. Latency is a concern for communications via GEO: for voice telephony this can be a problem but is less important for general data / internet and broadcast services. Our specific mission concept has significant requirements for on-orbit robotics and autonomy (both for the Servicer and the Tug). The interface with the Recycler is demanding: in addition to the requirements noted above (standard interfaces and modularity, guaranteed performance and availability) VASANT would be helped by having access to more components on-orbit (e.g. the thermal sub-system, antennas usable at microwave frequencies).

However, the major concern raised so far is the feasibility of the VASANT business model. Simply to launch a 100 tonne spacecraft to GEO will cost around $\$ 5 \mathrm{~B}$ using conventional launchers $\left(\$ 50 \mathrm{k} \mathrm{kg}^{-1}\right)$. A major effort is needed to reduce the VASANT mass and its construction, launch, and running costs. The same economic pressure applies to the Scavenger and Recycler: their products should be cost-effective relative to the costs of terrestrial manufacture plus launch.

In terms of strict sustainability (e.g. no increase in on-orbit mass), the engineering needed seems just about feasible now, and with developments in on-orbit robotics is becoming increasingly practical. However, the most cost-effective way of achieving strict sustainability currently seems to be to pay extra (about the same to de-orbit mass as to launch it in the first place) to dispose of unwanted mass from GEO by atmospheric re-entry. This is not a comfortable conclusion and does not feel right in terms of true sustainability, but it is where this (admittedly very early stage) study is currently pointing. To change this, significant developments are needed in

- Recyclability of on-orbit sub-systems and components (presumably requiring increased modularity) - especially to lower the cost of recycling; a whole system perspective will be needed so that scavenging, recycling, and reusing are conceived as a whole,

- Regulation, etc., of GEO, so that unsustainable activities are appropriately penalised. As a pre-cursor to this we suggest that more research is needed to understand what operational guidelines and regulation would lead to ways of using GEO which are truly sustainable (and that in turn probably requires work to achieve consensus about what sustainable really does mean for the GEO region). 
The question of orbit control in GEO is still open and could be significantly transformed if a way of creating propellant from on-orbit "waste" were available. One concept for this is to somehow pulverise old spacecraft parts into fine dust (initial calculations suggest this should be sub-nm in size) so that the dust particles can be accelerated electrostatically.

\section{FINAL REMARKS}

In this article we have proposed an on-orbit ecosystem and a scenario for sustainability in the GEO region. We do not claim that this scenario is complete or comprehensive, but it does allow us to explore the concept of recycling and reusing spacecraft parts. We have not considered the resilience of this concept to known but currently unpredictable threats (e.g. severe damage to spacecraft by micrometeoroids, debris collisions, or extreme solar weather). However, we do believe that sustainability along the lines we discuss is becoming technically feasible and this provides an inspiring challenge for the space industry.

As a summary of our findings, some features highlighted by these studies are (a) the technical challenges of reusing parts from old satellites: modularity and design-for-reuse seem to be key enablers, (b) the advanced robotics and autonomy implied by the on-orbit operations, (c) the challenge of long-term orbit control without excessive propellant consumption, and (d) there are major non-technical challenges for the business case, the legal use of debris, liability for accidents, and compliance with applicable regulations.

An indirect benefit of this study has been the involvement of around 40 postgraduate students (the three project teams). We have benefitted from their work and creativity, and they in turn have been exposed to some of the challenges which they will face during their careers in the space industry over the next few decades.

Many in the space sector are working to make our use of space sustainable. Nature requires us to achieve this eventually, and we offer this study as another step towards that goal.

\section{ACKNOWLEDGEMENTS}

This paper is based on the work performed by students on the Cranfield MSc in Astronautics and Space Engineering, academic year 2020-21. We gratefully acknowledge the contributions of the following students across the three project teams:

Debris Scavenger Spacecraft: Nick Brain, Irina-Stefana Bulgaru, Alexander Farr, Alexis Hudelle, Sam Juson, Guillem Khairy, Cameron Leslie, Ricardo Lozano Ortega, Andrej Misejka, Raffaele Pastore, Garance Petorin, Jesus Ramirez, Romain Steiner.

Recycler Space Station: Showy Chen, Edouard Demers, Tanvi Divkar, Sebastien Giafferi, Xingxing Liang, Ibrahima Mbaye, Sundeep Patel, Marco Rigamonti, Ariel Ropert, Ilias Sigalas, Dominic Thomas, Agathe Victor, Alexanda Whitworth-Biehler.

VASANT: Sakshi Baliyan, Jack Bowden, Maria Carrillo Barrenechea, Ramon Gonzalez Parrilla, Manfred Hamburgo Fragoso, Manohar Joel, Hillary Kamanzi , Kefei Lei, Hector Lleo, Jaime Martin Lozano, Nikita Shetti.

More details of these projects are available via the corresponding author on request.

\section{REFERENCES}

[1] Innocenti, L., Soares, T., Delaval, J., ESA Clean Space Initiative, 6th European Conference on Space Debris, Proceedings of the conference held 22-25 April 2013, in Darmstadt, Germany. Edited by L. Ouwehand. ESA SP723, ISBN 978-92-9221-287-2, 2013, id.4

[2] Towards the Circular Economy: an economic and business rationale for an accelerated transition. Ellen MacArthur Foundation. 2012.

[3] Barbara, N.H., Lizy-Destrez, S., Guardabasso, P., Alary, D., New GEO paradigm: Re-purposing satellite components from the GEO graveyard, (2020) Acta Astronautica, 173, pp. 155-163. https://doi.org/10.1016/j.actaastro.2020.03.041 
[4] Barnhart, D., Sullivan, B., Hunter, R., Bruhn, J., Fowler, E., Hoag, L., Chappie, S., Henshaw, G., Kelm, B., Kennedy, T., Mook, M., Vincent, K. Phoenix project status 2013, (2013) AIAA SPACE 2013 Conference and Exposition, https://doi.org/10.2514/6.2013-5341

[5] Stuttard, M., et al., Vast Satcom Antennas (VASANTs) as an enabling technology for commercial satellite communications. BIS Reinventing Space Conference, 2018

[6] Brain, Nicholas, Debris Scavenger Spacecraft: Target Capture and Component Extraction, Group project report, MSc in Astronautics and Space Engineering, Cranfield University, 2021.

[7] X. Zhu, I. C. Smith, F. Babin, "A hybrid 3D sensor (NEPTEC TriDAR) for object tracking and inspection," Proc. SPIE 6214, Laser Radar Technology and Applications XI, 621407 (19 May 2006); https://doi.org/10.1117/12.664716.

[8] Leslie, Cameron, Debris Scavenger Spacecraft: Attitude \& Orbit Control, Relative Navigation and Propulsion, Group project report, MSc in Astronautics and Space Engineering, Cranfield University, 2021.

[9] Juson, Sam, Mission Analysis \& Design, Proximity Operations, Group project report, MSc in Astronautics and Space Engineering, Cranfield University, 2021.

[10]Lleo, Hector, Advanced Comsat for Sustainable GEO: team management, requirements and budgets. Group project report, MSc in Astronautics and Space Engineering, Cranfield University, 2021.

[11] Hamburgo Fragoso, Manfred, Advanced Comsat for Sustainable GEO: Space Tug. Group project report, MSc in Astronautics and Space Engineering, Cranfield University, 2021. 
$2021-10-12$

\section{What might sustainability of the GEO region look like?}

Kingston, Jenny

Society of Photo-Optical Instrumentation Engineers (SPIE)

Kingston J, Felicetti L, Hobbs S. (2021) What might sustainability of the GEO region look like?.

In: Space, Satellites + Sustainability (S3), 28 September - 1 October 2021, Glasgow, UK, Volume 11888

https://doi.org/10.1117/12.2600964

Downloaded from Cranfield Library Services E-Repository 